\title{
Quantifying the Relationship between the Climate and Hand-Foot-Mouth Disease (HFMD) Incidences
}

\author{
Pei Fung Leong ${ }^{\# 1}$, Jane Labadin ${ }^{\# 2}$, Shapiee Bin Abd Rahman ${ }^{\# 3}$, Sarah Flora Samson Juan ${ }^{\# 4}$ \\ ${ }^{\#}$ Department of Computational Science and Mathematics, \\ Universiti Malaysia Sarawak, \\ 94300 Kota Samarahan, Sarawak, \\ Malaysia \\ 1peifung11@hotmail.com, \\ ${ }^{2}$ ljane@fit.unimas.my \\ ${ }^{3}$ sar@fit.unimas.my \\ 4sjsflora@fit.unimas.my
}

\begin{abstract}
This paper aims to examine the relationship between climate changes and Hand-Foot-Mouth Disease (HFMD) incidences. A study to examine the relationship between the weather and the HFMD cases in Sarawak, Malaysia had been done. However, the weather data obtained from the five meteorological stations in Sarawak were too few to represent the whole Sarawak, which account for unreliable results. To reach a better understanding of the effect of climate changes on the transmission of HFMD, this paper aims to quantify the relationship between climate changes and HFMD incidences in Singapore instead of Sarawak due to the smaller area of Singapore and the similar weather conditions for Singapore and Sarawak. The weather variables and incidences of HFMD during the period 2006-2008 had been collected on a weekly basis. The correlation analysis was conducted between weather variables and HFMD cases. Air temperature, pressure and wind speed were found to be significantly correlated with the number of HFMD cases in Singapore. This shows that weather variations may affect the transmission of HFMD in Singapore. It is suggested that air temperature could be used as a predictor of the number of HFMD cases in tropical countries. Thus, public health interventions should be conducted during this stage in order to adapt and overcome the possible affects of climate change in the future.
\end{abstract}

Keywords — relationship, climate, weather, Hand-Foot-Mouth Disease, HFMD

\section{INTRODUCTION}

Hand, foot and mouth disease (HFMD) is a common illness that affects mainly infants and children. A major outbreak of hand, foot and mouth disease in Sarawak, Malaysia in 1997 marked the beginning of a series of outbreaks in the Asia Pacific region [1]. From April through June 1997, 29 previously healthy children (with median age of 1.5 years) in Sarawak died of rapidly progressive cardiorespiratory failure during an outbreak of HFMD caused primarily by enterovirus 71 (EV71) [2]. A cluster of unusual paediatric deaths due to encephalitis and cardiac failure was observed in an outbreak of HEV71 in 1997 in Sarawak, a state of Malaysia on the island of Borneo [2, 3]. According to the sentinel surveillance programme conducted by the Sarawak Health Department and Institute of Health and Community Medicine, Universiti Malaysia Sarawak (UNIMAS), HFMD outbreaks has occurred every three years in Sarawak - 1997, 2000, 2003 and 2006 [1].

HFMD is transmitted through direct contact with nose discharge, saliva, feces and fluid from the blisters.
Additionally, it can spread through food and water which have been contaminated with fecal droplets or liquid from the mouth or nose of infected persons. Weather variables may affect the transmission of HFMD in both directly and indirectly way. The rate of replication of viruses and the survival of viruses in the environment may directly be affected by the surrounding temperature. Climate changes may affect the human behavior and hence influence the dynamics of HFMD. HFMD occurs during the summer in temperate countries but it occurs at any time during the year in the tropical countries [1]. Health experts expect the number of HFMD cases to rise as the weather warms. However, there are limited studies that discuss the associations between weather and the dynamics of HFMD in tropical countries. Instead of undertaking experiments to study the relationship between the weather and HFMD in the real world, we undertake experiments on mathematical representations of the real world as it would be costly to conduct in real life due to the time, risks and expenses involved. In this study, an investigation is carried out to reveal the relationship between weather and HFMD in Singapore.

Infectious diseases can have devastating impacts on human life and welfare [4]. Infectious diseases kill more than 14 million people, over a quarter of all deaths worldwide. It has also progressed to become the leading cause of suffering and mortality in many of the developing countries. By understanding the transmission characteristics of infectious diseases in communities, the transmission of infectious disease can be reduced. Thus far, research works that has been done for HFMD were mainly on clinical aspects of the disease. [5] and [6] researched on Human Enterovirus 71 (HEV71) in Taiwan while [7] studied HEV71 in Singapore and [8] studied HFMD in general in Singapore. Studies done by [9] and [4] on HFMD in Sarawak were also focused on HEV71. [10] did a phylogenetic analysis in Western Australia, Singapore and Malaysia regarding HEV71. [11] revealed that transmission coefficient and the immunity play vital roles in understanding the dynamics of HFMD. [12] analyzed the association between the weather and the occurrence of enterovirus complicated severe cases in Taiwan. The model was based on transformation function of seasonal factors in order to determine the transmission coefficient. [13] attempted to establish nonlinear mathematical models in order to simulate the incremental effects of global warming on HFMD incidences in Tokyo. These two models tried to find the relationship between the outbreaks of HFMD with the weather 
patterns in the respective countries. Similarly, this work will also find the relationship based on the weather patterns in Singapore.

A previous study [14] had been done to investigate the relationship between weather variables and HFMD incidences in Sarawak. However, the weather data for five meteorological stations in Sarawak were unable to represent the whole Sarawak as Sarawak is the largest state in Malaysia. The area of Sarawak is $124450 \mathrm{~km}^{2}$. Therefore, the result is questionable. In order to get a more reliable result in proving the relationship, this paper aims to quantify the relationship between climate changes and HFMD in Singapore. The area of Singapore is $710.2 \mathrm{~km}^{2}$, which is 175 times smaller than Sarawak. Based on the location of Singapore and Sarawak near the equator, the climates of Singapore and Sarawak are categorized as equatorial, being hot and humid throughout the year. Malaysia, in the south east part of Asia, has a geographic coordinate that reads $2^{\circ} 30^{\prime}$ North latitude and $112^{\circ} 30^{\prime}$ East longitude. Sarawak, which is the largest state of Malaysia, is located in between $2^{\circ} 0^{\prime}$ North latitude and $113^{\circ} 0^{\prime}$ East longitude. Singapore is located at the southern tip of the Peninsula Malaysia and lies in very close vicinity of the equator, which is located between $1^{\circ} 22^{\prime}$ North latitude and $103^{\circ} 48^{\prime}$ East longitude. The distance between Singapore and Kuching, Sarawak is 740.93 kilometres (460.39 miles). As a result of the latitude and longitude, both Sarawak and Singapore stay ahead by eight hours from the Greenwich Mean Time.

In order to examine the relationship between weather variables and HFMD, this study quantifies the association between the number of HFMD cases and weather variables, including air temperature, relative humidity, rainfall, and air pressure in Singapore from 2006 to 2008 using correlation analysis for existing disease surveillance data with the number of HFMD cases.

\section{METHODS}

\section{A. Background}

Singapore is an island country with total population of 5.08 million as at end-June 2010, of which about $17.4 \%$ under 15 years, $73.7 \%$ was $15-64$ years and $9.0 \%$ was 65 years of age and older [15]. The area of Singapore is $710.2 \mathrm{~km}^{2}$ with a density population of $7,022 / \mathrm{km}^{2}$ [15]. Singapore has a tropical rainforest climate having with no distinctive seasons, uniform temperature and pressure, high humidity, and abundant rainfall. Air temperatures range from 22 to $34{ }^{\circ} \mathrm{C}$. The lowest and highest air temperatures recorded in its maritime history are $19.4^{\circ} \mathrm{C}$ and $36^{\circ} \mathrm{C}[16]$.

\section{B. Data Collection}

1) Surveillance data: HFMD is endemic in Singapore. It became legally notifiable on 1st October 2000. Weekly notified cases of HFMD were retrieved from Ministry of Health Singapore $(\mathrm{MOH})$, the official organization publishing the notifiable disease data [17]. Since the date, medical practitioners are required to notify $\mathrm{MOH}$ for new cases of HFMD. In order to avoid under-reporting, a counter checking mechanism is in place to monitor and check for discrepancies.

2) Meteorological data: Hourly weather data consists of weekly pressure, relative humidity, wind speed and rainfall for Singapore was retrieved from the National University Singapore (NUS) Weather Station. The station is located on the rooftop of building E2 (Faculty of Engineering) of the National University of Singapore, Kent Ridge campus, at a height of about $90 \mathrm{~m}$ above sea level. The geographical coordinates are approximately: $1 \mathrm{deg} 18 \mathrm{~min} \mathrm{~N}$ (latitude) and 103 deg 46 min E (longitude). These data is chosen because it is the first weather station in Singapore to provide real-time meteorological data for free to students and the general public [18].

Although the record of notified HFMD cases in Singapore is from 2001, the study period in this work is from 2006 to 2008, due to some missing data from the NUS Weather Station before 2006 may influence the reliability of the result.

\section{Data Analysis}

The statistical relationship between weather data and incidences of HFMD in Singapore was assessed by correlation analysis. Having more than one independent variable is useful when predicting the dynamics of HFMD, as weather are all likely to be influenced by some combination of several factors. A set of independent variables can be tested precisely and knowing which variable is most influencing the transmission of HFMD. In this paper, the relationship between HFMD and weather is investigated. Variables such as pressure, air temperature, relative humidity, wind speed, and rainfall might contribute towards the transmission of HFMD. By analyzing all the available weather parameters against the HFMD incidences, we may be able to identify which of these weather parameters that show significant effect to the transmission of HFMD.

Correlation is a common and useful statistical parameter. A correlation is a single number that describes the degree of relationship between two variables. Knowing the score on one variable will be able to predict the score on the other variable if the two variables are correlated. The stronger the correlation, the closer the scores will fall to the regression line and hence the more accurate the prediction. A correlation coefficient is a number between -1 and 1 which measures the degree to which two variables are linearly related. If there is perfect linear relationship with positive slope between the two variables, we have a correlation coefficient of 1 ; if there is positive correlation, whenever one variable has a high (low) value, so does the other. If there is a perfect linear relationship with negative slope between the two variables, we have a correlation coefficient of -1 . This means with negative correlation, whenever one variable has a high (low) value, the other has a low (high) value. A correlation coefficient of 0 means that there is no linear relationship between the two variables investigated. The closer the number is to 1 or -1 , the stronger the correlation, or the stronger the relationship between the variables. The closer the number is to 0 , the weaker the correlation.

\section{RESULTS}

During the study period (2006-2008), there were a total of 65,050 HFMD cases in Singapore. Cases were from all age group with most cases from children below the age of four. Figure 1-5 shows the time series of the number of HFMD cases and weather variables (mean air temperature, pressure, relative humidity, wind speed and rainfall). It is observed that there was an outbreak every year. Table 1 shows the weather data when the number of HFMD is low (week 5), medium (week 73) and high (week 122) throughout 2006-2008. It is observed that the number of cases increases when the 